\title{
EXISTENCE OF SOLUTIONS FOR A MODEL OF SELF-GRAVITATING PARTICLES WITH EXTERNAL POTENTIAL
}

\author{
ANDRZEJ RACZYŃSKI \\ Instytut Matematyczny, Uniwersytet Wrocławski \\ Pl. Grunwaldzki 2/4, 50-384 Wroctaw, Poland \\ E-mail: Andrzej.Raczynski@math.uni.wroc.pl
}

\begin{abstract}
We study the existence of solutions to a nonlinear parabolic equation describing the temporal evolution of a cloud of self-gravitating particles with a given external potential. The initial data are in spaces of (generalized) pseudomeasures. We prove existence of local and global-in-time solutions, and also a kind of stability of global solutions.
\end{abstract}

1. Introduction. Let us consider the Cauchy problem for the equation

$$
u_{t}=\Delta u+\nabla \cdot(u \nabla \phi)+\nabla \cdot(u \nabla \Phi)
$$

coupled with the Poisson equation

$$
\nabla \phi=\nabla E_{d} * u
$$

where $E_{d}(z)=-\left((d-2) \sigma_{d}\right)^{-1}|z|^{2-d}, d \geq 3$, denotes the fundamental solution of the Laplacian in $\mathbb{R}^{d}$, and $\sigma_{d}$ is the area of the unit sphere in $\mathbb{R}^{d}$. Moreover, in (1), $\Phi$ is a given function.

We supplement system (1)-(2) with the initial condition

$$
u(x, 0)=u_{0}(x) .
$$

The system above describes temporal evolution of the density $u(x, t)$ of a cloud of self-gravitating particles and the potential $\phi(x, t)$ generated by gravitational interaction between them. The function $\Phi(x)$ appearing in the third term on the right-hand side of (1) represents the given external potential.

The physical (astrophysical) interpretation of the system has a long history and, to find more details, we refer the reader to the papers [1]-[3] and references therein.

2000 Mathematics Subject Classification: 35K15, 35B40, 82C21.

Key words and phrases: nonlinear parabolic-elliptic system, Cauchy problem, pseudomeasure, external potential.

The paper is in final form and no version of it will be published elsewhere. 
The paper was inspired by the paper [4] where the system above with no external potential was considered.

Notations. We denote the Fourier transform of a function $v$ on $\mathbb{R}^{d}$ by the symbol $\widehat{v}(\xi)=$ $\int_{\mathbb{R}^{d}} v(x) e^{-i x \cdot \xi} d x$. The constant $C$ denotes various inessential constants and may vary from line to line.

The spaces $\mathcal{P} \mathcal{M}^{d-\beta}$. Following [4], we introduce functional Banach spaces relevant to the study of solutions of the Cauchy problem for the system (1)-(3):

$$
\mathcal{P} \mathcal{M}^{a}=\left\{v \in \mathcal{S}^{\prime}\left(\mathbb{R}^{d}\right): \widehat{v} \in L_{\text {loc }}^{1}\left(\mathbb{R}^{d}\right),\|v\|_{\mathcal{P M}^{a}} \equiv \underset{\xi \in \mathbb{R}^{d}}{\operatorname{ess} \sup }|\xi|^{a}|\widehat{v}(\xi)|<\infty\right\}
$$

where $a \geq 0$. More properties of these spaces can be found in [4].

Let us define, following [4], the space of vector-valued functions $\mathcal{X}_{\beta}$ as follows:

$$
\mathcal{X}_{\beta}=\mathcal{C}_{w}\left([0, T] ; \mathcal{P M}^{d-\beta}\right), \quad 0<T \leq \infty,
$$

where $\mathcal{C}_{w}$ denotes (cf. [1]) the space of vector-valued functions which are weakly continuous. The necessity of considering $\mathcal{C}_{w}$ instead of strongly continuous functions is caused by the fact that the heat semigroup is only weakly continuous on the spaces $\mathcal{P} \mathcal{M}^{a}$, since these spaces are not separable.

By a solution of the system (1)-(3) we understand a function $u=u(t) \in \mathcal{X}_{\beta}$ fulfilling the equation

(4) $\widehat{u}(\xi, t)=e^{-t|\xi|^{2}} \widehat{u}(\xi, 0)+\int_{0}^{t} e^{-(t-s)|\xi|^{2}} i \xi \cdot\left(\widehat{u}(\xi, s) *\left(\frac{i \xi}{|\xi|^{2}} \widehat{u}(\xi, s)+\widehat{\nabla \Phi}(\xi, s)\right)\right) d s$

for $\xi \in \mathbb{R}^{d}$ and $0 \leq t<T$.

Recall that a mild solution of problem (1)-(3) is defined usually as a solution of the following integral equation:

$$
u(t)=e^{t \Delta} u_{0}+\int_{0}^{t} e^{(t-s) \Delta} \nabla \cdot(u(s) \nabla \phi(s)) d s+\int_{0}^{t} e^{(t-s) \Delta} \nabla \cdot(u(s) \nabla \Phi(s)) d s,
$$

where $\nabla \phi(s)=\nabla E_{d} * u(s)$, and the integral is understood as the Bochner integral. Such a meaning of a solution is not suitable for our construction of solutions of the Cauchy problem. The integrals with respect to $s$ in equations (4) and (5) should be defined in a weak sense (like, for example, in [4] and [10, Def. 2]). For more explanations, we refer the reader to [4] and references therein. Nevertheless, a distributional solution of (1)-(3) which belongs to $\mathcal{X}_{\beta}$ is the solution of the integral equation (4) (or its equivalent version (5)), and vice versa. This equivalence can be proved following the computations for Navier-Stokes equations in [10, Th. 5.2].

The spaces $\mathcal{P} \mathcal{M}^{d-2}$ and $\mathcal{X}_{2}$ play a special role in our considerations. This comes from the fact that for $d \geq 3$ there exists a stationary solution to the problem with no external potential. It is called the Chandrasekhar solution and has the form

$$
u_{C}(x)=2(d-2)|x|^{-2} \text {. }
$$

It is easy to check that $u_{C}(x)$ belongs to $\mathcal{P} \mathcal{M}^{d-2}$ (and to $\mathcal{X}_{2}$ if $u_{C}$ is interpreted as a constant function in $t$ ). As it was shown in [4], it is expected that $u_{C}$ is a solution with a kind of critical singularity of initial data in the sense that for small $\left(\leq \varepsilon u_{C}\right)$ initial data 
the solution exists, and for an initial condition such that $u_{0}(x)>u_{C}$ there is no solution to problem (1)-(3).

In this paper we use also the space $\mathcal{P} \mathcal{M}^{d-\beta}, \beta<2$. A typical example of a function which belongs to $\mathcal{P} \mathcal{M}^{d-\beta}$ is $|x|^{-\beta}$.

The model (1)-(3) can be also considered with the electric interaction instead of the gravitational one; for a physical motivation see [8], [9]. In this case the equation (2) should be rewritten as

$$
\nabla \phi=-\nabla E_{d} * u \text {. }
$$

However, the results for this model are usually "better" than for gravitational one, but the methods used for construction of solutions here do not allow obtain different results. Thus the results we can obtain for the electric interactions model are the same (as well as the proofs) as for the model considered in this paper.

To simplify the notation, we will denote the quadratic term in (4) or (5) by $\widehat{B}(u, u)$ or $B(u, u)$ resp., with the bilinear form $B$ defined by

$$
B(u, v)=\int_{0}^{t} e^{(t-s) \Delta} \nabla \cdot(u(s) \nabla \phi(s)) d s,
$$

where $\phi(s)$ is obtained by $\nabla \phi(s)=\nabla E_{d} * v(s)$.

Analogously, let us denote the linear term in (4) or (5) by $L u$ or $\widehat{L} u$, respectively, with $L$ defined by

$$
L u=\int_{0}^{t} e^{(t-s) \Delta} \nabla \cdot(u(s) \nabla \Phi(s)) d s
$$

where $\Phi$ is a given external potential such that $\nabla \Phi \in \mathcal{P} \mathcal{M}^{d-\alpha}$.

This paper is the first one where such a condition on the function $\Phi$ is imposed. A usual condition for the external potential was $\Phi=q^{*} E_{d}$, cf. [8]. Unfortunately, such a singular form of the function $\Phi$ implies that the operator $L$ cannot be well-defined. $\nabla \Phi \in \mathcal{P} \mathcal{M}^{d-\alpha}$ is a condition which allows us to define the operator $L$ properly.

So our problem can be read as

$$
u(t)=e^{t \Delta} u_{0}+B(u, u)+L u .
$$

2. Main tools. The main tool which will be used in the paper is a modification of a well-known theorem by Banach, that gives the existence and uniqueness of solutions via a contraction mapping (or successive approximations) argument. We have (cf. [7])

Theorem 2.1. Let $B: \mathcal{X} \times \mathcal{X} \rightarrow \mathcal{X}$ be a continuous bilinear operator. Assume that there exists a constant $K$ such that

$$
\|B(y, z)\|_{\mathcal{X}} \leq K\left\|_{y}\right\|_{\mathcal{X}}\|z\|_{\mathcal{X}}
$$

for all $y, z \in \mathcal{X}$. Let $L: \mathcal{X} \rightarrow \mathcal{X}$ be a continuous linear operator

$$
\|L y\|_{\mathcal{X}} \leq \ell\|y\|_{\mathcal{X}}
$$

with the norm satisfying the condition $\ell<1$.

i) For $a \in \mathcal{X}$ such that $\|a\|_{\mathcal{X}} \leq \frac{(1-\ell)^{2}}{4 K}$, there exists a solution $x \in \mathcal{X}$ to the equation

$$
x=a+L x+B(x, x) .
$$


This solution fulfills the estimate

$$
\|x\|_{\mathcal{X}} \leq \frac{1-\ell-\sqrt{(1-\ell)^{2}-4 K\|\|_{\mathcal{X}}}}{2 K} \leq \frac{1-\ell}{2 K} .
$$

Additionally, this solution is unique in the open ball in $\mathcal{X}$ centered at zero, of radius $\frac{1-\ell}{2 K}$. ii) The solution depends continuously on a in the following way. For $\|b\|_{\mathcal{X}} \leq \varepsilon<\frac{(1-\ell)^{2}}{4 K}$ and $v$ being the solution of the equation $v=b+L v+B(v, v)$, we have

$$
\|u-v\|_{\mathcal{X}} \leq 2\left((1-\ell)^{2}-4 K \varepsilon\right)^{-\frac{1}{2}}\|a-b\|_{\mathcal{X}} .
$$

The proof, following the lines of [7, Ch. 16, Lemma 20], is left to the reader.

In the proofs we will often use the following estimate of the integral $\int_{0}^{t} e^{-(t-s)|\xi|^{2}} d s$. LEMMA 2.2 .

$$
\int_{0}^{t} e^{-(t-s)|\xi|^{2}} d s=\frac{1-e^{-t|\xi|^{2}}}{|\xi|^{2}} \leq q^{q} \frac{t^{1-q}}{|\xi|^{2 q}}, \quad \text { for every } q \in[0,1] .
$$

Proof. Since for $q=0$ (we put $0^{0}=1$ ) and $q=1$ the proof is obvious, hence we assume $0<q<1$. Putting $q=1-\frac{1}{p}$, it suffices to show the inequality

$$
\frac{1-e^{-t|\xi|^{2}}}{\left(t|\xi|^{2}\right)^{\frac{1}{p}}} \leq\left(1-\frac{1}{p}\right)^{1-\frac{1}{p}}, \quad \text { for every } 1<p<+\infty .
$$

However, estimating the maximal value of the function $f(z)=\frac{1-e^{-z^{p}}}{z}$ we have that this maximal value is reached for $z=z_{\max }$. Moreover, $z_{\max }$ fulfills the equation $e^{-z_{\max }^{p}}=$ $\frac{1}{1+p z_{\max }^{p}}$. Thus

$$
f\left(z_{\max }\right)=\frac{p z_{\max }^{p}}{p z_{\max }^{p+1}+z_{\max }} \leq\left(1-\frac{1}{p}\right)^{1-\frac{1}{p}}=q^{q}, \quad q \in[0,1] .
$$

Let us recall the formula for the Fourier transform for the function $|x|^{-\alpha}$ :

$$
\left(\widehat{|x|^{-\alpha}}\right)=C|\xi|^{\alpha-d} \quad \text { for every } 0<\alpha<d
$$

and the formula for convolution of such functions for $0<\alpha, \beta<d$, such that $d<\alpha+\beta$ :

$$
|x|^{-\alpha} *|x|^{-\beta}=C_{\alpha, \beta}|x|^{d-\alpha-\beta},
$$

where (following [4, Lemma 2.1]) $C_{\alpha, \beta}$ is equal to

$$
C_{\alpha, \beta}=\pi^{d / 2} \frac{\Gamma\left(\frac{d-\alpha}{2}\right) \Gamma\left(\frac{d-\beta}{2}\right) \Gamma\left(\frac{\alpha+\beta-d}{2}\right)}{\Gamma\left(\frac{\alpha}{2}\right) \Gamma\left(\frac{\beta}{2}\right) \Gamma\left(\frac{2 d-\alpha-\beta}{2}\right)},
$$

with $\Gamma$ denoting the usual Gamma function.

3. Global and local solutions in the space $\mathcal{X}_{\beta}$. In this section we prove the existence of local (in the space $\mathcal{X}_{\beta}, \beta<2$ ) and global (in the space $\mathcal{X}_{2}$ ) solutions to the problem, using Theorem 2.1 and suitable estimates of the bilinear form $B(\cdot, \cdot)$ and operator $L$.

First, let us observe that if $u_{0} \in \mathcal{P} \mathcal{M}^{d-\beta}$ then $e^{t \Delta} u_{0} \in \mathcal{P} \mathcal{M}^{d-\beta}$ for every $t>0$, but in general $e^{t \Delta} u_{0} \notin \mathcal{P} \mathcal{M}^{d-\gamma}$ for $\gamma \neq \beta$. Indeed, we have

$$
\left\|e^{t \Delta} u_{0}\right\|_{\mathcal{X}_{\beta}}=\sup _{0 \leq t \leq T} \operatorname{ess} \sup _{\xi \in \mathbb{R}^{d}}|\xi|^{d-\beta} e^{-t|\xi|^{2}}\left|\widehat{u_{0}}\right| \leq\left\|u_{0}\right\|_{\mathcal{P M}^{d-\beta}}=\left\|u_{0}\right\|_{\mathcal{X}_{\beta}}
$$


To get estimates for $\|B(u, u)\|_{\mathcal{X}_{\beta}}$ let us notice that if $v(t) \in \mathcal{P} \mathcal{M}^{d-\beta}$ and $\nabla \psi(t)=$ $\nabla E_{d} * v(t)$ then $\widehat{\nabla \psi}(\xi, t)=i \xi|\xi|^{-2} \hat{v}(\xi, t)$ so $\nabla \psi(t) \in \mathcal{P} \mathcal{M}^{d-\beta+1}$. Thus, we have

LemMA 3.1. For $1<\beta<\frac{d+1}{2}, \beta \leq 2$ and functions $u, v \in \mathcal{X}_{\beta}$ we have

$$
\|B(u, v)\|_{\mathcal{X}_{\beta}} \leq K\|u\|_{\mathcal{X}_{\beta}}\|v\|_{\mathcal{X}_{\beta}}
$$

where

$$
K=C_{d-\beta, d-\beta+1}\left(\frac{\beta}{2}\right)^{\frac{\beta}{2}} T^{1-\frac{\beta}{2}} .
$$

with $C_{d-\beta, d-\beta+1}$ defined by (10).

Proof. For $u(t) \in \mathcal{P} \mathcal{M}^{d-\beta}$ and $d$ and $\beta$ fulfilling the inequality

$$
1<\beta<\frac{d+1}{2}
$$

we have

$$
\begin{aligned}
|(\hat{u}(t) * \widehat{\nabla \psi}(t))(\xi)| & \leq\left(\int_{\mathbb{R}^{d}}|\xi-\zeta|^{\beta-d}|\zeta|^{\beta-1-d} d \zeta\right)\|u(t)\|_{\mathcal{P M}^{d-\beta}}\|v(t)\|_{\mathcal{P M}^{d-\beta}} \\
& =|\xi|^{2 \beta-1-d} C_{d-\beta, d-\beta+1}\|u(t)\|_{\mathcal{P M}^{d-\beta}}\|v(t)\|_{\mathcal{P M}^{d-\beta}} .
\end{aligned}
$$

Thus, for $u, v \in \mathcal{X}_{\beta}$, using Lemma 2.2 we obtain

$$
\begin{aligned}
\|B(u, v)\|_{\mathcal{X}_{\beta}} & =\sup _{0 \leq t \leq T} \operatorname{ess~sup}_{\xi \in \mathbb{R}^{d}}|\xi|^{d-\beta}\left|\int_{0}^{t} i \xi e^{-(t-s)|\xi|^{2}} \widehat{(u \nabla \psi)}(s) d s\right| \\
& \leq \sup _{0 \leq t \leq T} \underset{\xi \in \mathbb{R}^{d}}{\operatorname{ess~sup}}|\xi|^{\beta} C_{d-\beta, d-\beta+1} \int_{0}^{t} e^{-(t-s)|\xi|^{2}} d s\|u\|_{\mathcal{X}_{\beta}}\|v\|_{\mathcal{X}_{\beta}} \\
& =C_{d-\beta, d-\beta+1} q^{q} T^{1-q}|\xi|^{\beta-2 q}\|u\|_{\mathcal{X}_{\beta}}\|v\|_{\mathcal{X}_{\beta}} \quad \text { for every } q \in[0,1] .
\end{aligned}
$$

To get the required estimate for the norm of $\|B(u, u)\|_{\mathcal{X}_{\beta}}$, we put $q=\frac{\beta}{2}$, hence $1-q=1-\frac{\beta}{2} \geq 0$.

Proving the existence of global solutions we need additionally $q=1\left(=\frac{\beta}{2}\right)$. In the case of local existence, it is enough that $1-q=1-\frac{\beta}{2}>0$, which implies $\beta<2$. Thus the constant $K$ required in Theorem 2.1 is equal to

$$
K=C_{d-\beta, d-\beta+1}\left(\frac{\beta}{2}\right)^{\frac{\beta}{2}} T^{1-\frac{\beta}{2}},
$$

and for $\beta=2$ the constant does not depend on $T$.

We have an analogous result for the operator $L$.

Lemma 3.2. For $\alpha, \beta>0, \alpha \leq 1, \alpha+\beta<d$, $\Phi$ such that $\nabla \Phi \in \mathcal{P M}^{d-\alpha}$ and function $u \in \mathcal{X}_{\beta}$ we have

$$
\|L u\|_{\mathcal{X}_{\beta}} \leq \ell\|u\|_{\mathcal{X}_{\beta}}
$$

where

$$
\ell=\|\nabla \Phi\|_{\mathcal{P M}^{d-\alpha}} C_{d-\beta, d-\alpha}\left(\frac{\alpha+1}{2}\right)^{\frac{\alpha+1}{2}} T^{\frac{1-\alpha}{2}}
$$


Proof. For the exponents $\alpha, \beta$ satisfying

$$
\alpha, \beta>0, \quad \alpha+\beta<d,
$$

we have

$$
\begin{aligned}
|(\hat{u}(t) * \widehat{\nabla \Phi}(t))(\xi)| & \leq\left(\int_{\mathbb{R}^{d}}|\xi-\zeta|^{\beta-d}|\zeta|^{\alpha-d} d \zeta\right)\|\nabla \Phi\|_{\mathcal{P M}^{d-\alpha}}\|u(t)\|_{\mathcal{P M}^{d-\beta}} \\
& =|\xi|^{\beta+\alpha-d} C_{d-\beta, d-\alpha}\|\nabla \Phi\|_{\mathcal{P M}^{d-\alpha}}\|u(t)\|_{\mathcal{P M}^{d-\beta}} .
\end{aligned}
$$

Thus we have

$$
\begin{aligned}
\|L u\|_{\mathcal{X}_{\beta}} & =\sup _{t \geq 0} \underset{\xi \in \mathbb{R}^{d}}{\operatorname{esssup}}|\xi|^{d-\beta}\left|\int_{0}^{t} i \xi e^{-(t-s)|\xi|^{2}} \widehat{(u \nabla \Phi)}(s) d s\right| \\
& \leq \sup _{t \geq 0} \underset{\xi \in \mathbb{R}^{d}}{\operatorname{essup}} C_{d-\beta, d-\alpha}|\xi|^{\alpha+1}\left(\int_{0}^{t} e^{-(t-s)|\xi|^{2}} d s\right)\|u\|_{\mathcal{X}_{\beta}}\|\nabla \Phi\|_{\mathcal{P M}^{d-\alpha}} \\
& =\sup _{t \geq 0} \underset{\xi \in \mathbb{R}^{d}}{\operatorname{essup}} q^{q} C_{d-\beta, d-\alpha}|\xi|^{\alpha+1-2 q} T^{1-q}\|u\|_{\mathcal{X}_{\beta}}\|\nabla \Phi\|_{\mathcal{P M}^{d-\alpha}} .
\end{aligned}
$$

Hence to obtain the finite norm $\|L u\|_{\mathcal{X}_{\beta}}$ we should take $q=\frac{\alpha+1}{2}$, so, $1-q=\frac{1-\alpha}{2} \geq 0$.

To get a global solution we need $\alpha=1$, but to obtain local existence it is enough to take $\alpha<1$. Note that the conditions of existence in global and local case do not depend on $\beta$.

So for such $q=\frac{\alpha+1}{2}$ and with the assumption $\alpha \leq 1$ we have an estimate for

$$
\ell=\|\nabla \Phi\|_{\mathcal{P M}^{d-\alpha}} C_{d-\beta, d-\alpha}\left(\frac{\alpha+1}{2}\right)^{\frac{\alpha+1}{2}} T^{\frac{1-\alpha}{2}} .
$$

The required condition $\ell<1$ (needed in Theorem 2.1) reads

$$
\|\nabla \Phi\|_{\mathcal{P M}^{d-\alpha}}<C_{d-\beta, d-\alpha}^{-1}\left(\frac{\alpha+1}{2}\right)^{-\frac{\alpha+1}{2}} T^{-\frac{1-\alpha}{2}} .
$$

It is interesting to observe that for $\alpha=1$ we can satisfy the condition above taking $\|\nabla \Phi\|_{\mathcal{P M}^{d-\alpha}}$ small enough only. But for $\alpha<1$ we can get the same result taking $T$ small enough and any value of $\|\nabla \Phi\|_{\mathcal{P M}^{d-\alpha}}$. So for $\alpha<1$ local existence can be proved for arbitrary values of external potential. This is impossible for $\alpha=1$ using our method.

Now we are ready to prove the following theorem.

Theorem 3.3. Assume that $u_{0} \in \mathcal{P} \mathcal{M}^{d-\beta}$ and $\Phi$ satisfies $\nabla \Phi \in \mathcal{P} \mathcal{M}^{d-\alpha}$. Considering the equation

$$
u=e^{t \Delta} u(0)+L u+B(u, u)
$$

we have

i) (global-in-time solution) for $\beta=2, \alpha=1, d \geq 4, \Phi$ such that $\|\nabla \Phi\|_{\mathcal{P M}^{d-1}}<$ $C_{d-2, d-1}^{-1}$, and $u_{0} \in \mathcal{P} \mathcal{M}^{d-2}$ satisfying

$$
\left\|u_{0}\right\|_{\mathcal{P M}^{d-2}}<\frac{\left(1-\|\nabla \Phi\|_{\mathcal{P M}^{d-1}} C_{d-2, d-1}\right)^{2}}{4 C_{d-2, d-1}},
$$

there exists a unique global-in-time solution to the problem (16) (we take $T=+\infty$ in the definition of $\mathcal{X}_{2}$ ), 
ii) (local-in-time solution for $\alpha=1$ ) for $d \geq 3, \alpha=1,1<\beta<2$, $\Phi$ such that $\|\nabla \Phi\|_{\mathcal{P M}^{d-1}}<C_{d-\beta, d-1}^{-1}$, all values of $\left\|u_{0}\right\|_{\mathcal{P M}^{d-\beta}}$ and $T$ small enough, there exists a local-in-time solution to the problem (16),

iii) (local-in-time solution for $d=3$ ) for $d \geq 3,0<\alpha<1,1<\beta<2$, all values of $\left\|u_{0}\right\|_{\mathcal{P M}^{d-\beta}},\|\nabla \Phi\|_{\mathcal{P M}^{d-\alpha}}$ and $T$ small enough, there exists a local-in-time solution to the problem (16).

Proof. $i$ ) To prove global existence we should take $\beta=2$ (so the space considered is $\mathcal{X}_{2}$ with $T=+\infty)$ and $\alpha=1$ (to get the constants $K(12)$ and $\ell$ (14) independent of time $T$ ). Additionally, due to (11) (and (13)), we should assume $d \geq 4$. For such constants, $\|\nabla \Phi\|_{\mathcal{P M}^{d-\alpha}}<C_{d-2, d-1}^{-1}$ and $\left\|u_{0}\right\|_{\mathcal{P M}^{d-2}}$ fulfilling

$$
\left\|u_{0}\right\|_{\mathcal{P M}^{d-2}} \leq \frac{\left(1-\|\nabla \Phi\|_{\mathcal{P M}^{d-1}} C_{d-2, d-1}\right)^{2}}{4 C_{d-2, d-1}}
$$

we apply Theorem 2.1 to get the required result. The proof of the weak continuity (in this and in the next two cases) follows the lines of the argument in [7, Ch. 18, Lemma 24] or [10, Th. 3.1].

ii) Taking $\alpha=1$ we get $l$ independent of time (see (14)). But for $1<\beta<2$ (and the space $\mathcal{X}_{\beta}$ with $T$ small enough, chosen later) we can get the local existence of the solution for all values of $\left\|u_{0}\right\|_{\mathcal{P M}^{d-\beta}}$. In fact, for $\|\nabla \Phi\|_{\mathcal{P M}^{d-1}}<C_{d-\beta, d-1}^{-1}$ and $T$ small enough the condition

$$
\left\|u_{0}\right\|_{\mathcal{P M}^{d-\beta}}<\frac{\left(1-\|\nabla \Phi\|_{\mathcal{P M}^{d-1}} C_{d-\beta, d-1}\right)^{2}}{4 C_{d-\beta, d-\beta+1}\left(\frac{\beta}{2}\right)^{\frac{\beta}{2}} T^{1-\frac{\beta}{2}}}
$$

is fulfilled for all $\left\|u_{0}\right\|_{\mathcal{P M}^{d-\beta}}$, which guarantees that the assumptions of Theorem 2.1 are satisfied.

iii) In fact, we can also prove existence of a local solution not only for arbitrary values of $\left\|u_{0}\right\|_{\mathcal{P M}^{d-\beta}}$ but also for arbitrary values of $\|\nabla \Phi\|_{\mathcal{P M}^{d-\alpha}}$. We can prove it taking $\alpha<1$. Indeed the norm must fulfill (due to Theorem 2.1) the condition

$$
\left\|u_{0}\right\|_{\mathcal{P M}^{d-\beta}}<\frac{(1-\ell)^{2}}{4 K}=\frac{\left(1-\|\nabla \Phi\|_{\mathcal{P M}^{d-\alpha}} C_{d-\beta, d-\alpha}\left(\frac{\alpha+1}{2}\right)^{\frac{\alpha+1}{2}} T^{\frac{1-\alpha}{2}}\right)^{2}}{4 C_{d-\beta, d-\beta+1}\left(\frac{\beta}{2}\right)^{\frac{\beta}{2}} T^{1-\frac{\beta}{2}}} .
$$

For a given value of $\|\nabla \Phi\|_{\mathcal{P M}^{d-\alpha}}>0$ we take $T$ small enough, such that the condition (15) is satisfied. Since the right-hand side of the inequality (17) tends to $+\infty$ as $T$ tends to 0 , so taking $T$ small enough (and smaller than the one chosen in (15)), we can satisfy the above inequality for all values of $\left\|u_{0}\right\|_{\mathcal{P M}^{d-\beta}}$ and $\|\nabla \Phi\|_{\mathcal{P M}^{d-\alpha}}$.

4. Existence of solutions in the new space $\mathcal{Y}_{\beta}$. The existence of global solutions has not been proved for the three-dimensional case yet. We will do it in a subspace of $\mathcal{X}_{2}$, i.e. in a subspace of functions belonging to $\mathcal{X}_{2}$ with some control in time of the norm $\|u(t)\|_{\mathcal{P M}^{d-\beta}}$.

Define

$$
\mathcal{Y}_{\beta}=\left\{v(t) \in \mathcal{S}^{\prime}\left(\mathbb{R}^{d}\right): \widehat{v}(t) \in L_{\mathrm{loc}}^{1}\left(\mathbb{R}^{d}\right) \text { for a.e. } t,\|v\|_{\mathcal{Y}_{\beta}}=\sup _{t>0} t^{1-\frac{\beta}{2}}\|v(t)\|_{\mathcal{P M}^{d-\beta}}\right\}
$$

where $\beta \geq 0$. 
The norm in the space $\mathcal{X}_{2} \cap \mathcal{Y}_{\beta}$ is given by

$$
\|v\|_{\mathcal{X}_{2} \cap \mathcal{Y}_{\beta}}=\|v\|_{\mathcal{X}_{2}}+\|v\|_{\mathcal{Y}_{\beta}} .
$$

The first question is: which space does $e^{t \Delta} u_{0}$ belong to? From the previous section we know that $u_{0} \in \mathcal{P} \mathcal{M}^{d-\beta}$ implies $e^{t \Delta} u_{0} \in \mathcal{X}_{\beta}$.

Considering the spaces $\mathcal{Y}_{\beta}$ we can prove that $u_{0} \in \mathcal{P M}^{d-2}$ implies $e^{t \Delta} u_{0} \in \mathcal{Y}_{\beta}, \beta \leq 2$. Moreover, the importance of the space $\mathcal{P M}^{d-2}$ follows from the fact that for $\beta \leq 2$ $e^{t \Delta} u_{0} \in \mathcal{Y}_{\beta}$ necessitates that $u_{0} \in \mathcal{P M}^{d-2}$. Indeed we have

LEMma 4.1. For any $u_{0} \in \mathcal{P M}^{d-2}$ the solution of the heat equation $e^{t \Delta} u_{0}$ belongs to $\mathcal{Y}_{\beta}$ for $\beta \leq 2$.

Proof. The statement of the lemma is a consequence of the following calculations:

$$
\begin{aligned}
\left\|e^{t \Delta} u_{0}\right\| \mathcal{Y}_{\beta} & =\sup _{t>0} \underset{\xi \in \mathbb{R}^{d}}{\operatorname{essup} t^{1-\frac{\beta}{2}}}|\xi|^{d-\beta} e^{-t|\xi|^{2}}\left|\hat{u}_{0}\right| \\
& =\sup _{t>0} \underset{\xi \in \mathbb{R}^{d}}{\operatorname{ess} \sup }\left(t|\xi|^{2}\right)^{1-\frac{\beta}{2}} e^{-t|\xi|^{2}}|\xi|^{d-2}\left|\hat{u}_{0}\right| \leq\left(1-\frac{\beta}{2}\right)^{1-\frac{\beta}{2}} e^{-\left(1-\frac{\beta}{2}\right)}\left\|u_{0}\right\|_{\mathcal{P M}^{d-2}}
\end{aligned}
$$

for $\beta \leq 2$ and $u_{0} \in \mathcal{P} \mathcal{M}^{d-2}$. The norm of $e^{t \Delta} u_{0}$ is equal to

$$
\left\|e^{t \Delta} u_{0}\right\|_{\mathcal{X}_{2} \cap \mathcal{Y}_{\beta}}=\left(1+\left(1-\frac{\beta}{2}\right)^{1-\frac{\beta}{2}} e^{-\left(1-\frac{\beta}{2}\right)}\right)\left\|u_{0}\right\|_{\mathcal{P M}^{d-2}} .
$$

To prove the existence of solutions in the space $\mathcal{X}_{2} \cap \mathcal{Y}_{\beta}, \beta<2$, we must estimate $B(u, v)$ and $L u$ in $\mathcal{P M}^{d-2}$ and $\mathcal{Y}_{\beta}$. The following lemmas give the required inequalities.

Lemma 4.2. For $1<\beta<d-1$, the functions $u \in \mathcal{X}_{2}$ and $v \in \mathcal{Y}_{\beta}$ we have

$$
\|B(u, v)\|_{\mathcal{X}_{2}} \leq C\|u\|_{\mathcal{X}_{2}}\|v\|_{\mathcal{Y}_{\beta}},
$$

where

$$
C=C_{d-2, d-\beta+1}\left(\frac{\beta}{2}\right)^{\frac{\beta}{2}}\left(1+e^{-\frac{\beta}{2}} \frac{2}{\beta}\right) .
$$

Proof. Analogously as in the previous section we have

$$
|\widehat{u \nabla \phi}(t)| \leq C_{d-2, d-\beta+1}|\xi|^{\beta+1-d}\|u(t)\|_{\mathcal{P M}^{d-2}}\|v(t)\|_{\mathcal{P M}^{d-\beta}},
$$

hence

$$
\begin{aligned}
\|B(u, v)\|_{\mathcal{X}_{2}} & =\sup _{t>0} \underset{\xi \in \mathbb{R}^{d}}{\operatorname{esssup}} C_{d-2, d-\beta+1}|\xi|^{\beta}\left|\int_{0}^{t} e^{-(t-s)|\xi|^{2}} s^{\frac{\beta}{2}-1} d s\right|\|u\|_{\mathcal{X}_{2}}\|v\|_{\mathcal{Y}_{\beta}} \\
& \leq C\|u\|_{\mathcal{X}_{2}}\|v\|_{\mathcal{Y}_{\beta}},
\end{aligned}
$$

where

$$
C=C_{d-2, d-\beta+1}\left(\frac{\beta}{2}\right)^{\frac{\beta}{2}}\left(1+e^{-\frac{\beta}{2}} \frac{2}{\beta}\right) .
$$

To prove the last inequality we consider two cases:

- For $\beta \geq 2$ we have (due to (7))

$$
|\xi|^{\beta} \int_{0}^{t} e^{-(t-s)|\xi|^{2}} s^{\frac{\beta}{2}-1} d s \leq|\xi|^{\beta} t^{\frac{\beta}{2}-1} \frac{t^{1-q}}{|\xi|^{2 q}} q^{q}=q^{q}\left(t|\xi|^{2}\right)^{\frac{\beta}{2}-q}, \quad q \in[0,1] .
$$


Taking $q=\frac{\beta}{2}$ we get

$$
\|B(u, v)\|_{\mathcal{X}_{2}} \leq C_{d-2, d-\beta+1}\left(\frac{\beta}{2}\right)^{\frac{\beta}{2}}\|u\|_{\mathcal{X}_{2}}\|v\|_{\mathcal{Y}_{\beta}} .
$$

- For $1<\beta<2$ we have

$$
\begin{aligned}
|\xi|^{\beta} \int_{0}^{t} e^{-(t-s)|\xi|^{2}} s^{\frac{\beta}{2}-1} d s & \leq|\xi|^{\beta} e^{-\frac{t}{2}|\xi|^{2}} \int_{0}^{\frac{t}{2}} s^{\frac{\beta}{2}-1} d s+|\xi|^{\beta}\left(\frac{t}{2}\right)^{\frac{\beta}{2}-1} \int_{\frac{t}{2}}^{t} e^{-(t-s)|\xi|^{2}} d s \\
& \leq\left(\frac{t}{2}|\xi|^{2}\right)^{\frac{\beta}{2}} e^{-\frac{t}{2}|\xi|^{2}} \frac{2}{\beta}+q^{q}\left(\frac{t}{2}|\xi|^{2}\right)^{\frac{\beta}{2}-q} \cdot
\end{aligned}
$$

Thus, taking the maximal value of the function $z^{\frac{\beta}{2}} e^{-z}$ in the first term, and putting $q=\frac{\beta}{2}$ in the second one, we get for $1<\beta<2$

$$
\|B(u, v)\|_{\mathcal{X}_{2}} \leq C_{d-2, d-\beta+1}\left(\frac{\beta}{2}\right)^{\frac{\beta}{2}}\left(1+e^{-\frac{\beta}{2}} \frac{2}{\beta}\right)\|u\|_{\mathcal{X}_{2}}\|v\|_{\mathcal{Y}_{\beta}} .
$$

So for $d \geq 3$ and $1<\beta<d-1$, we obtain the required constant $C$ as was stated in the Lemma.

Following the preceding reasoning we can prove the estimate

Lemma 4.3. For $1<\beta<d-1$, the functions $u \in \mathcal{X}_{2}$ and $v \in \mathcal{Y}_{\beta}$ we have

$$
\|B(u, v)\|_{\mathcal{Y}_{\beta}} \leq C\|u\|_{\mathcal{X}_{2}}\|v\|_{\mathcal{Y}_{\beta}}
$$

where

$$
C=C_{d-2, d-\beta+1} 2^{1-\frac{\beta}{2}}\left(1+\frac{2}{\beta} e^{-1}\right)
$$

Next we prove estimates for $L u$. From the previous section we know that for $\alpha=1$ and $0<\beta<d-1$ we have $\|L u\|_{\mathcal{X}_{\beta}} \leq C\|u\|_{\mathcal{X}_{\beta}}$.

Lemma 4.4. For $1<\beta<d-1$, $\Phi$ such that $\nabla \Phi \in \mathcal{P M}^{d-1}$ and the function $u \in \mathcal{Y}_{\beta}$ we have

$$
\|L u\|_{\mathcal{X}_{2}} \leq C\|u\|_{\mathcal{Y}_{\beta}},
$$

where

$$
C=C_{d-\beta, d-1}\left(\frac{\beta}{2}\right)^{\frac{\beta}{2}}\left(1+e^{-\frac{\beta}{2}} \frac{2}{\beta}\right)\|\nabla \Phi\|_{\mathcal{P M}^{d-1}}
$$

Proof. We have

$$
\begin{aligned}
|\widehat{u \nabla \Phi}| & \leq \int_{\mathbb{R}^{d}}|\hat{u}(\xi-\zeta)||\zeta|^{1-d} d \zeta\|\nabla \Phi\|_{\mathcal{P M}^{d-1}} \\
& \leq C_{d-\beta, d-1}|\xi|^{\beta+1-d}\|u(t)\|_{\mathcal{P M}^{d-\beta}}\|\nabla \Phi\|_{\mathcal{P M}^{d-1}}
\end{aligned}
$$

Thus

$\|L u\|_{\mathcal{X}_{2}}=\left.\sup _{t>0} \operatorname{ess~sup}_{\xi \in \mathbb{R}^{d}}|\xi|^{d-2}\left|\int_{0}^{t} e^{-(t-s)|\xi|^{2}} i \xi C_{d-\beta, d-1}\right| \xi\right|^{\beta+1-d}\|u(s)\|_{\mathcal{P M}^{d-\beta}} d s \mid\|\nabla \Phi\|_{\mathcal{P M}^{d-1}}$ 


$$
\begin{array}{r}
\leq C_{d-\beta, d-1}\|u\|_{\mathcal{Y}_{\beta}}\|\nabla \Phi\|_{\mathcal{P M}^{d-1}} \sup _{t>0} \operatorname{ess~sup}_{\xi \in \mathbb{R}^{d}}|\xi|^{\beta} \int_{0}^{t} e^{-(t-s)|\xi|^{2}} s^{\frac{\beta}{2}-1} d s \\
\leq C_{d-\beta, d-1}\left(\frac{\beta}{2}\right)^{\frac{\beta}{2}}\left(1+e^{-\frac{\beta}{2}} \frac{2}{\beta}\right)\|\nabla \Phi\|_{\mathcal{P M}^{d-1}}\|u\|_{\mathcal{Y}_{\beta}} .
\end{array}
$$

The proof of the last inequality can be rewritten from Lemma 4.2 where such an estimate for $\int_{0}^{t} e^{-(t-s)|\xi|^{2}} s^{\frac{\beta}{2}-1} d s$ appears.

It can be easily seen that for $\alpha \neq 1$ the integral is unbounded.

Analogously we can prove

Lemma 4.5. For $d \geq 3,1<\beta \leq 2$, $\Phi$ such that $\nabla \Phi \in \mathcal{P M}^{d-1}$ and the function $u \in \mathcal{Y}_{\beta}$ we have

$$
\|L u\|_{\mathcal{Y}_{\beta}} \leq C\|u\|_{\mathcal{Y}_{\beta}}
$$

where

$$
C=C_{d-2, d-1}\left(\frac{\beta}{2}\right)^{\frac{\beta}{2}}\|\nabla \Phi\|_{\mathcal{P M}^{d-1}}
$$

To prove existence of a global solution we apply Lemmas $4.2,4.3$ to get the estimate

$$
\|B(u, v)\|_{\mathcal{X}_{2} \cap \mathcal{Y}_{\beta}} \leq K\|u\|_{\mathcal{X}_{2} \cap \mathcal{Y}_{\beta}}\|v\|_{\mathcal{X}_{2} \cap \mathcal{Y}_{\beta}} .
$$

Then applying Lemmas 4.4, 4.5 we get

$$
\|L u\|_{\mathcal{X}_{2} \cap \mathcal{Y}_{\beta}} \leq \ell\|u\|_{\mathcal{X}_{2} \cap \mathcal{Y}_{\beta}} .
$$

These inequalities allow us to apply Theorem 2.1 to obtain existence and uniqueness of global solutions. Thus we arrive at

TheOREM 4.6. Let $d \geq 3$ and $1<\beta<2$. For $\Phi$ such that $\nabla \Phi \in \mathcal{P M}^{d-1}$ with $\|\nabla \Phi\|_{\mathcal{P M}^{d-1}}$ small enough (such that $\left.\ell=\ell\left(\|\nabla \Phi\|_{\mathcal{P M}^{d-1}}\right)<1\right)$, and $\left\|u_{0}\right\|_{\mathcal{P M}^{d-2}}$ such that

$$
\left\|u_{0}\right\|_{\mathcal{P M}^{d-2}}<\frac{(1-\ell)^{2}}{4 K}
$$

with constants $K$ and $\ell$ obtained from (18) and (19), there exists a unique solution $u \in$ $\mathcal{X}_{2} \cap \mathcal{Y}_{\beta}$ to the problem (6).

A simple consequence of the above theorem is the following proposition:

Proposition 4.7. For $d \geq 4$ there is no stationary solution $U=U(x)$ to the problem (1)-(3) with small norm $\|U\|_{\mathcal{P M}^{d-2}}$.

Proof. Let us assume that such a stationary solution exists. Since its norm $\|U\|_{\mathcal{P M}^{d-2}}$ is small enough, we can obtain a unique global solution belonging to $\mathcal{X}_{2}$ (due to Theorem 3.3 ) and to its subspace $\mathcal{X}_{2} \cap \mathcal{Y}_{\beta}, \beta<2$ (due to Theorem 4.6). From Theorem 3.3 we conclude that $U$ must be this global solution (but independent of time). But due the uniqueness and Theorem 4.6 $U$ must belong also to $\mathcal{Y}_{\beta}$. This implies that $t^{1-\frac{\beta}{2}}\|U\|_{\mathcal{P M}^{d-2}}$ must be bounded what is imposible. So stationary solution with small norm in $\mathcal{P M}^{d-2}$ cannot exist. 
REMARK. The next possibility of proving the existence of solutions to the problem is to consider a more general space $\mathcal{Y}_{\gamma, \beta}$ equipped with the norm

$$
\|u\|_{\mathcal{Y}_{\gamma, \beta}}=\sup _{t>0} \operatorname{ess} \sup _{\xi \in \mathbb{R}^{d}} t^{\gamma}\|u(t)\|_{\mathcal{P M}^{d-\beta}}
$$

Unfortunately, the condition $u_{0} \in \mathcal{P} \mathcal{M}^{d-2}$ implies $e^{t \Delta} u_{0} \in \mathcal{Y}_{\gamma, \beta}$ only for $\gamma=1-\frac{\beta}{2}$. This means that $e^{t \Delta} u_{0} \in \mathcal{Y}_{\beta}\left(=\mathcal{Y}_{1-\frac{\beta}{2}, \beta}\right)$. It is obvious since the norm of $e^{t \Delta} u_{0}$ is equal to

$$
\begin{aligned}
\left\|e^{t \Delta} u_{0}\right\| \mathcal{Y}_{\gamma, \beta} & =\sup _{t>0} \underset{\xi \in \mathbb{R}^{d}}{\operatorname{ess} \sup } t^{\gamma}|\xi|^{d-\beta} e^{-t|\xi|^{2}}\left|\hat{u}_{0}\right| \\
& =\sup _{t>0} \underset{\xi \in \mathbb{R}^{d}}{\operatorname{essup}}\left(t|\xi|^{2}\right)^{1-\frac{\beta}{2}} e^{-t|\xi|^{2}}|\xi|^{d-2}\left|\hat{u}_{0}\right| t^{\gamma-1+\frac{\beta}{2}}
\end{aligned}
$$

which is unbounded for $\gamma \neq 1-\frac{\beta}{2}$.

REMARK. We can ask whether it is possible to repeat the construction of global solution in $\mathcal{X}_{\beta} \cap \mathcal{Y}_{\gamma, \delta}, \delta<\beta$ (as in Theorem 4.6), but with $u_{0} \in \mathcal{P M}^{d-\beta}, \beta<2$ (let us remind that for such $u_{0}$ we get only a local solution). Unfortunately, it fails for two reasons. The first one is that $\left\|e^{t \Delta} u_{0}\right\|_{\mathcal{Y}_{\gamma, \delta}} \leq C\left\|u_{0}\right\|_{\mathcal{X}_{\beta}}$ only for $\gamma=\frac{\beta-\delta}{2}$. The second one is that $\|B(u, v)\|_{\mathcal{X}_{\beta}} \leq C\|u\|_{\mathcal{X}_{\beta}}\|v\|_{\mathcal{Y}_{\gamma, \delta}}$ with $C$ independent of time holds true only for $\gamma=1-\frac{\delta}{2}$ what implies that $\beta$ must be equal to 2 .

5. Asymptotic stability of solutions. In this section we would like to prove convergence of solutions to the evolution problem towards stationary states. Due to Proposition 4.7 such a solution with a small norm cannot exist. Thus we prove in this section the asymptotic stability of solutions. The first observation is that if we take $u(t)$, the solution with $u_{0} \in \mathcal{P} \mathcal{M}^{d-2}$, and $v(t)$, the solution of the heat equation with the same initial condition, we have (due the estimates for $\|B(u, v)\|_{\mathcal{P M}^{d-2}}$ and $\|L u\|_{\mathcal{P M}^{d-2}}$ )

$$
\|u(t)-v(t)\|_{\mathcal{P M}^{d-2}} \leq\|B(u, u)\|_{\mathcal{P M}^{d-2}}+\|L u\|_{\mathcal{P M}^{d-2}} \leq K\|u\|_{\mathcal{X}_{2}}^{2}+\ell\|u\|_{\mathcal{X}_{2}} \leq \text { const. }
$$

This means that the solutions $u(t)$ stay in a neighbourhood of $v(t)$.

For $\beta=2$ (we restrict to the case $\beta=2$ since we have a global solution only in the space $\mathcal{X}_{2}$ ), we are able only to prove following result concerning asymptotic stability.

ThEOREM 5.1. Let $u(t)$ and $v(t)$ be the solutions of the problem with initial data $u(0)$ and $v(0)$ respectively, and the same external potential $\Phi$. We take $u(0)$ and $v(0)$ such that $\|u(0)\|_{\mathcal{P M}^{d-2}} \leq \varepsilon<\frac{(1-\ell)^{2}}{4 K}$ and $\|v(0)\|_{\mathcal{P M}^{d-2}} \leq \varepsilon<\frac{(1-\ell)^{2}}{4 K}$. If, additionally, the solutions of the heat equation with the same initial conditions $u(0), v(0)$ approach each other, i.e.

$$
\lim _{t \rightarrow+\infty}\left\|e^{t \Delta}(u(0)-v(0))\right\|_{\mathcal{P M}^{d-2}}=0
$$

then also

$$
\lim _{t \rightarrow+\infty}\|u(t)-v(t)\|_{\mathcal{P M}^{d-2}}=0 .
$$

Proof. First, let us estimate the integral

$$
\sup _{\xi \in \mathbb{R}^{d}} \int_{0}^{t}|\xi|^{2} e^{-(t-s)|\xi|^{2}}\|f(s)\|_{\mathcal{P M}^{d-2}} d s .
$$


For $p>1$ and $\frac{1}{q}=1-\frac{1}{p}$ we have

$$
\begin{aligned}
& \sup _{\xi \in \mathbb{R}^{d}} \int_{0}^{t}|\xi|^{2} e^{-(t-s)|\xi|^{2}}\|f(s)\|_{\mathcal{P M}^{d-2}} d s \\
& \quad \leq \sup _{\xi \in \mathbb{R}^{d}} \frac{q}{t} \int_{0}^{\frac{t}{p}} \frac{t|\xi|^{2}}{q} e^{-\frac{t}{q}|\xi|^{2}}\|f(s)\|_{\mathcal{P M}^{d-2}} d s+\sup _{\xi \in \mathbb{R}^{d}}|\xi|^{2} \frac{1-e^{-\frac{t}{q}|\xi|^{2}}}{|\xi|^{2}} \sup _{\frac{t}{p} \leq s \leq t}\|f(s)\|_{\mathcal{P M}^{d-2}} \\
& \quad \leq\left(\frac{p}{e(p-1) t}\right) \int_{0}^{\frac{t}{p}}\|f(s)\|_{\mathcal{P M}^{d-2}} d s+\sup _{\frac{t}{p} \leq s \leq t}\|f(s)\|_{\mathcal{P M}^{d-2}} \\
& \quad \leq\left(\frac{p}{e(p-1)}\right) \int_{0}^{\frac{1}{p}}\|f(t s)\|_{\mathcal{P M}^{d-2}} d s+\sup _{\frac{t}{p} \leq s \leq t}\|f(s)\|_{\mathcal{P M}^{d-2} .} .
\end{aligned}
$$

Coming back to the main conclusion, we have

$$
\begin{aligned}
\|u(t)-v(t)\|_{\mathcal{P M}^{d-2}} \leq & \left\|e^{t \Delta}(u(0)-v(0))\right\|_{\mathcal{P M}^{d-2}}+\|B(u-v, u)\|_{\mathcal{P M}^{d-2}} \\
& +\|B(v, u-v)\|_{\mathcal{P M}^{d-2}}+\|L(u-v)\|_{\mathcal{P M}^{d-2}} \\
\leq & \left\|e^{t \Delta}(u(0)-v(0))\right\|_{\mathcal{P M}^{d-2}} \\
& +2 \max \left\{\|u\|_{\mathcal{X}_{2}},\|v\|_{\mathcal{X}_{2}}\right\} K \sup _{\xi \in \mathbb{R}^{d}} \int_{0}^{t}|\xi|^{2} e^{-(t-s)|\xi|^{2}}\|(u-v)(s)\|_{\mathcal{P M}^{d-2}} d s \\
& +\ell \sup _{\xi \in \mathbb{R}^{d}} \int_{0}^{t}|\xi|^{2} e^{-(t-s)|\xi|^{2}}\|(u-v)(s)\|_{\mathcal{P M}^{d-2}} d s \\
\leq & \left\|e^{t \Delta}(u(0)-v(0))\right\|_{\mathcal{P M}^{d-2}}+\left(2 K \frac{1-\ell-\sqrt{(\ell-1)^{2}-4 K \varepsilon}}{2 K}+\ell\right) \\
& \times\left(\frac{p}{e(p-1)} \int_{0}^{\frac{1}{p}}\|(u-v)(t s)\|_{\mathcal{P M}^{d-2}} d s+\sup _{\frac{t}{p} \leq s \leq t}\|(u-v)(s)\|_{\mathcal{P M}^{d-2}}\right) \\
\leq & \left\|e^{t \Delta}(u(0)-v(0))\right\|_{\mathcal{P M}^{d-2}}+\left(1-\sqrt{(\ell-1)^{2}-4 K \varepsilon}\right) \\
& \times\left(\frac{p}{e(p-1)} \int_{0}^{\frac{1}{p}}\|(u-v)(t s)\|_{\left.\mathcal{P M}^{d-2} d s+\sup _{\frac{t}{p} \leq s \leq t}\|(u-v)(s)\|_{\mathcal{P M}^{d-2}}\right) .}\right.
\end{aligned}
$$

Since we want to calculate the $\operatorname{limit}_{\lim } \rightarrow+\infty\|u(t)-v(t)\|_{\mathcal{P M}^{d-2}}$, let us define

$$
A=\limsup _{t \rightarrow+\infty}\|u(t)-v(t)\|_{\mathcal{P M}^{d-2}}=\limsup _{t \geq k \in \mathbb{N}, k \rightarrow+\infty}\|u(t)-v(t)\|_{\mathcal{P M}^{d-2}} .
$$

Let us observe that, due to assumption (20) and the Lebesgue Dominated Convergence Theorem, the inequality above reads

$$
A \leq\left(\frac{1}{e(p-1)}+1\right)\left(1-\sqrt{(\ell-1)^{2}-4 K \varepsilon}\right) A .
$$

So if $\left(\frac{1}{e(p-1)}+1\right)\left(1-\sqrt{(\ell-1)^{2}-4 K \varepsilon}\right)<1$, which is equivalent to $\sqrt{(\ell-1)^{2}-4 K \varepsilon}>$ $\frac{1}{e(p-1)+1}$, then $A=0$.

Indeed, the value of the left-hand side of the last inequality belongs to the interval $(0,|1-\ell|), \ell<1$, since $0<\varepsilon<\frac{(1-\ell)^{2}}{4 K}$. Thus, taking $p>1$ great enough, we can get the right-hand size of the last inequality less than $\sqrt{(\ell-1)^{2}-4 K \varepsilon}$. This implies that $A=0$, i.e.

$$
\lim _{t \rightarrow+\infty}\|u(t)-v(t)\|_{\mathcal{P M}^{d-2}}=0
$$


REMARK. The condition (20) in the assumption of Theorem 5.1 is equivalent to

$$
\lim _{\xi \rightarrow 0}|\xi|^{d-2}\left|\widehat{u_{0}}(\xi)-\widehat{v_{0}}(\xi)\right|=0 .
$$

Indeed, for any $\varepsilon>0$ there exists $\delta=\delta(\varepsilon)$ such that for $|\xi|<\delta$ we have $|\xi|^{d-2} \mid \widehat{u_{0}}(\xi)-$ $\widehat{v_{0}}(\xi) \mid \leq \varepsilon$. So

$$
\underset{|\xi| \leq \delta}{\operatorname{ess} \sup } e^{-t|\xi|^{2}}|\xi|^{d-2}\left|\widehat{u_{0}}(\xi)-\widehat{v_{0}}(\xi)\right| \leq \varepsilon
$$

For $|\xi|>\delta$ we have

$$
\underset{|\xi| \geq \delta}{\operatorname{ess} \sup } e^{-t|\xi|^{2}}|\xi|^{d-2}\left|\widehat{u_{0}}(\xi)-\widehat{v_{0}}(\xi)\right| \leq e^{-t \delta^{2}}\left(\left\|u_{0}\right\|_{\mathcal{P M}^{d-2}}+\left\|v_{0}\right\|_{\mathcal{P M}^{d-2}}\right) \leq \varepsilon
$$

for $t=t\left(\delta, u_{0}, v_{0}\right)\left(=t\left(\varepsilon, u_{0}, v_{0}\right)\right)$ large enough. Thus

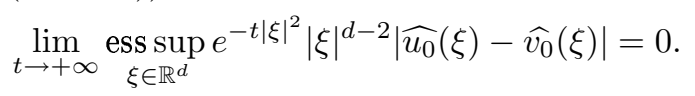

On the other hand, if the condition (21) is not fulfilled, then there exists a sequence $\left\{\xi_{k}\right\}, \xi_{k} \rightarrow 0$, such that the limit considered in (21) is equal to some $A>0$. So

$$
\underset{\xi \in \mathbb{R}^{d}}{\operatorname{ess} \sup } e^{-t|\xi|^{2}}|\xi|^{d-2}\left|\widehat{u_{0}}(\xi)-\widehat{v_{0}}(\xi)\right| \geq \lim _{\xi_{k} \rightarrow 0} e^{-t\left|\xi_{k}\right|^{2}}\left|\xi_{k}\right|^{d-2}\left|\widehat{u_{0}}\left(\xi_{k}\right)-\widehat{v_{0}}\left(\xi_{k}\right)\right|=A,
$$

where $A$ does not depend on $t$, and condition (20) cannot be satisfied.

Acknowledgements. The preparation of this paper was supported by the KBN grant 2/P03A/011/19. The author would like to express his thanks to Grzegorz Karch for discussions during the preparation of this paper.

\section{References}

[1] P. Biler, The Cauchy problem and self-similar solution for a nonlinear parabolic equation, Studia Math. 114 (1995), 181-205.

[2] P. Biler, Growth and accretion of mass in an astrophysical model, Appl. Math. (Warsaw) 23 (1995), 179-189.

[3] P. Biler, Existence and nonexistence of solutions for a model of gravitational interaction of particles, III, Colloq. Math. 68 (1995), 229-239.

[4] P. Biler, M. Cannone, I. A. Guerra and G. Karch, Global regular and singular solutions for a model of gravitating particles, Math. Ann. 330 (2004), 693-708.

[5] P.-H. Chavanis, J. Sommeria and R. Robert, Statistical mechanics of two-dimensional vortices and collisionless stellar systems, Astrophys. J. 471 (1996), 385.

[6] E. H. Lieb and M. Loss, Analysis, Second edition, AMS, Providence, RI, 2001.

[7] Y. Meyer, Wavelets, paraproducts and Navier-Stokes equations, 105-212, in: Current Developments in Mathematics, 1996, International Press, Cambridge, MA, 1999.

[8] I. Rubinstein, Electro-Diffusion of Ions, SIAM Studies in Appl. Math. 11, Philadelphia, 1990.

[9] I. Rubinstein and L. Rubinstein, Partial Differential Equations in Classical Mathematical Physics, Cambridge University Press, Cambridge, 1993.

[10] M. Yamazaki, The Navier-Stokes equations in the weak- $L^{n}$ spaces with time-dependent external force, Math. Ann. 317 (2000), 635-675. 